\title{
El discurso interpersonal en la guía turística en español y alemán y su importancia para la traducción
}

\author{
INTERPERSONAL DISCOURSE IN THE TOURISTIC GUIDE IN SPANISH AND GERMAN AND \\ ITS IMPORTANCE FOR TRANSLATION
}

\author{
Francisca Suau Jiménez y María Labarta Postigo \\ IULMA, Universitat de València
}

\section{RESUMEN:}

\begin{abstract}
El objetivo de este trabajo es el estudio contrastivo del discurso interpersonal del turismo a través de sus marcadores en el género "Guía Turística", y las implicaciones para la traducción español-alemán. Para ello se analiza un corpus piloto de 24.000 palabras que contiene tres bloques: textos en alemán y español como lengua original, y textos en alemán como lengua traducida. A partir del análisis contrastivo de cómo actúa la función interpersonal y los marcadores en ambas lenguas, en este género y en el área del turismo (Hyland 2005, Suau Jiménez, 2012), estudiamos si esta función se ha tenido en cuenta en la traducción al alemán, con objeto de evaluar si cumple con el principio de una traducción pragmática o de calidad (Nord 2007, Durán Muñoz 2012). Los resultados permiten constatar que ambas lenguas originales, español y alemán, presentan diferencias relevantes en cómo materializan el discurso interpersonal y la cantidad y calidad de sus marcadores. Sin embargo, el alemán como lengua traducida reproduce los marcadores del español y no tiene en cuenta la lengua meta y su correspondiente discurso interpersonal. Este uso interpersonal deficiente nos lleva a concluir que puede haber existido deterioro en la expresión de la persuasión, esencial en este género, el cual podría, como consecuencia, no cumplir bien su función promocional.
\end{abstract}

PALABRAS CLAVE: discurso interpersonal, marcadores, traducción español-alemán, turismo, función promocional 


\section{ABSTRACT:}

The purpose of this paper is to study the interpersonal discourse of tourism through its markers in the genre "Touristic Guide", and the implications for the translation Spanish-German, from a constrastive perspective. To this end, a pilot corpus of 24,000 words has been analyzed, with three different subcorpora: texts in German as original language, texts in Spanish as original language, and texts in German as translated language. We have analyzed how does the interpersonal discursive function work in this genre and in the sphere of tourism (Hyland, 2005; Suau Jiménez, 2012), by means of markers in both original languages. Then, the translated language has been scrutinized in order to evaluate whether it complies with the principle of pragmatic translation (Nord, 2007; Durán Muñoz, 2012), i.e. taking into account the interpersonal discourse in the target language of the translation. Results suggest that the texts in the original languages, Spanish and German, show relevant differences in the way in which they both materialize the interpersonal discourse, as well as the amount and kind of markers they use. However, German as a translated language reproduces the Spanish interpersonal discourse, not considering that of the target language. Therefore, it can be concluded that due to this interpersonal misuse, there may have been some damage in the expression of persuasion, essential for this genre, and therefore a certain limitation to attain its promotional function.

KEYWORDS: interpersonal discourse, markers, Spanish-German translation, tourism, promotional function

\section{LA GUÍA TURÍSTICA Y SU DISCURSO}

La Guía Turística es un género con una función promocional, que se realiza persuadiendo al cliente potencial para que elija un destino, un itinerario o unos productos y servicios concretos. En su evolución desde el formato impreso tradicional hasta el actual formato digital, sus elementos característicos no han variado en lo esencial. Tal como apuntan Santulli (2007) y Gallardo (2009), y recoge Calvi (2016), su rasgo más importante es la prescripción, -implícita en la propia denominación de este género-, que suele obedecer a una cierta concepción del turismo, según la institución pública o privada que haya detrás; otro de sus rasgos más representativos es la recomendación. Hay que mencionar igualmente, y de forma muy importante en español (Suau Jiménez 2012a), las expresiones impersonales, tales como verbos en infinitivo o terceras personas en modo reflexivo, que atenúan la mencionada fuerza prescriptiva y permiten una supuesta objetividad.

Cabe destacar, sin embargo, que con la explosión de los géneros en formato digital a través de internet, aparecen unos condicionantes discursivos nuevos que inciden en el producto lingüístico final. Por una parte, el inglés, como lengua franca internacional y como idioma dominante hasta ahora en el ámbito del turismo y sus instituciones, ejerce una cierta influencia en el discurso de la Guía Turística en cuanto a cómo se expresan las funciones retóricas 
prototípicas -prescripción y recomendación-, junto con otras que están al servicio de estas, tales como la descripción o la ejemplificación. Esta influencia también se da en la forma en la cual se relaciona el autor con el lector, es decir, en la relación interpersonal. Las guías turísticas se traducen a un buen número de lenguas desde el inglés y también desde otras lenguas, y esto puede provocar que se reproduzcan los modos y las estrategias discursivas interpersonales de la lengua origen (Durán Muñoz, 2012; Suau Jiménez, 2012b, 2015), lo cual puede dar lugar a un discurso "contaminado" que por no estar adaptado al uso lingüístico habitual y reconocido por los lectores de la lengua de llegada, puede ocasionar cierto daño al propio propósito comunicativo, la persuasión del lector y finalmente, la promoción. Por otra parte, el hecho de que el público en general, bajo la etiqueta de turistas, consumidores o usuarios, participe activamente en el fenómeno turístico, dando opiniones sobre productos y servicios a través de foros, blogs y redes sociales, ha propiciado una transformación del discurso de la Guía Turística. Tal como concluye Calvi (2016), este género se encuentra en un punto de cierta inflexión, ya que no responde a los usos lingüísticos tradicionales del antiguo formato impreso, pero tampoco acaba de cristalizar como género 2.0. En él se mezclan la promoción turística, la divulgación de conocimientos y la experiencia personal, a lo cual habría que añadir quizá la influencia de la función interpersonal, con marcadores y estrategias que conforman un diálogo implícito entre autor y lector, y que son el resultado de unas variables de lengua-cultura, de género y de especialidad, principalmente (Suau Jiménez, 2016). En otras palabras, los destinatarios de guías turísticas digitales han variado en sus expectativas, respecto a las antiguas guías impresas, que eran supuestamente imparciales y objetivas. Ahora esperan un discurso que contenga evaluaciones basadas en la experiencia como elemento de persuasión y de credibilidad, con lo cual el resultado es más subjetivo y también más narrativo, en una especie de hibridación genérica que discurre entre lo objetivo y lo subjetivo.

En el caso que nos ocupa, el discurso de la Guía Turística tiene unas características genéricas parecidas en alemán y en español, empezando por el propósito comunicativo. También sus funciones comunicativas son semejantes, tales como la descripción histórica, geográfica, cultural o artística, la explicación o la ejemplificación. Por otra parte, el registro incluirá un lenguaje desde formal-técnico hasta estándar y a veces coloquial, pudiéndose etiquetar de discurso de especialidad híbrido, por alternar tecnicismos y elementos coloquiales. Basado en el patrón lingüístico-cultural español o alemán, puede haber variación en el registro, que es donde se inserta la función interpersonal, ya sea con un claro contenido proposicional o de contenido, con abundantes adjetivos calificativos y adverbios de tono positivo para realzar las descripciones, como ocurre en el patrón español, o bien con abundantes elementos de tipo puramente metadiscursivo, tales como atenuadores, pronominalización, automención, etc., como ocurre en el patrón alemán, donde el contenido proposicional queda más matizado o modulado.

A continuación, exponemos dos ejemplos del patrón lingüístico-cultural alemán y del español, donde destacamos en cursiva los elementos del discurso interpersonal que propician la singularidad de cada lengua: 


\begin{tabular}{l}
\hline Deutschlandurlaub - alles und noch viel mehr! \\
\hline "Deutschland kann Ihnen viel bieten: Ob Urlaub mit Familie oder eine Reise aus beruflichen \\
Gründen, ob Kulturtrip oder Badeferien, ob Aktivurlaub oder sich einfach nur gesund \\
verwöhnen lassen - Deutschland kann Ihnen jeden Wunsch erfüllen (...)" \\
Lernen Sie die Geheimnisse der deutschen Städte kennen, entdecken Sie die romantischen \\
und die aufregenden Seiten Deutschlands, und lassen Sie sich von Land und Leuten \\
verzaubern!" \\
http://www.tiscover.de/de/guide/5de,de/objectId,RGN20de/home.html
\end{tabular}

En el caso del alemán se utilizan matizadores o atenuadores y marcadores de compromiso. En la primera oración del ejemplo, el verbo modal können (poder, con el sentido de probabilidad) en su función de atenuador, deja abierta a la imaginación del lector las muchas posibilidades (viel) que ofrece (anbieten) el destino turístico, en este caso Alemania: "Deutschland kann Ihnen viel anbieten". Veamos la segunda oración: "Deutschland kann Ihnen jeden Wunsch erfüllen" (Alemania le puede satisfacer (a usted) cualquier deseo). De nuevo a través del verbo modal können, el contenido proposicional queda más atenuado o matizado. En lugar de decir: "Venga usted a Alemania, aquí se le cumplirán todos sus deseos", el autor alemán transmite a través del können en su uso modal da la idea de probabilidad, de posibilidad y matiza el contenido. El discurso adquiere mayor fuerza de persuasión al introducir aquí además la referencia apelativa Ihnen (a usted). Cabe destacar que en alemán las referencias apelativas son mayoritariamente de uso formal, y se usan en la comunicación interpersonal cuando los interlocutores no se conocen. Según Nord (2002:159) esto refleja el uso de las formas de tratamiento en la vida real de esta cultura.

Aunque no aparecen aquí marcadores de actitud ni de automención, sí es abundante el uso de marcadores de compromiso, mediante el uso del imperativo. Solo en la última frase tenemos tres imperativos apelando a la segunda persona que es el lector -lernen Sie, entdecken Sie, lassen Sie sich...verzaubern!-. Aparece también en este caso la referencia apelativa directa a través de Sie, de tratamiento formal. El autor está constantemente apelando al lector de manera directa, con la finalidad de despertar su interés e integrarlo en el proceso comunicativo: lernen Sie (conozca usted); entecken Sie (descubra usted) lassen Sie sich...verzaubern (déjese hechizar, encantar).

En cuanto a los adjetivos calificativos y sustantivos escogidos, todos ellos son positivos o de connotación seductora: romantische, aufregende, die Geheimnisse der deutschen Städte, para atraer la atención del lector hacia los valores turísticos del destino.

Por último, resaltaremos el frecuente uso de partículas modales o marcadores del discurso, como por ejemplo "einfach nur" en el texto del ejemplo. Nord (2002) afirma en su trabajo que el alemán usa más partículas modales y marcadores del discurso que el español y que el inglés. 
"¿Qué hacer en Valencia?

Acercarse a la imponente Cartuja de Porta Coeli, contemplar las mejores obras de arte en el IVAM o en marzo, en las fiestas de Las Fallas, asistir a las espectaculares mascletaes de la Plaza del Ayuntamiento"

www.comunitatvalenciana.com

En el caso del español se puede observar cómo se utilizan los verbos en infinitivo para expresar recomendaciones, con un uso metadiscursivo distante y exento de marcadores que aludan directamente al lector. Así, la pregunta inicial de “¿Qué hacer en Valencia?”, que en sí es un marcador dirigido al lector/turista, es contestada por toda una serie de verbos en infinitivo, tales como "acercarse", "contemplar" o "asistir", también dirigidos al lector, pero sugiriendo diferentes acciones de forma distante. No se utiliza el imperativo, como sí ocurre en inglés debido a su trasfondo pragmático-cultural (Suau Jiménez, 2012b), que prescribe una comunicación más directa con el lector.

Así mismo, los adjetivos calificativos positivos son abundantes para realzar valores del turismo que interesa poner de relieve, y que de esta manera se objetivizan, tales como lo grandioso, lo mejor, lo espectacular (Mapelli, 2008; Pierini, 2009, Suau Jiménez 2012a). De esta manera, el autor intenta transmitir a través del discurso una sensación de credibilidad y autoridad sobre el contenido, como forma de persuadir al lector. Aquí no figuran tampoco marcadores de automención, ni de actitud, ni de compromiso -marcadores del lector-, estos últimos al contrario que en alemán, donde sí se utilizan, de forma similar a lo que ocurre en inglés (Suau Jiménez 2012b).

\section{EL TURISMO: DIFERENCIAS INTERCULTURALES Y EFECTO EN LA TRADUCCIÓN}

En comparación con los análisis realizados sobre otros tipos de textos especializados, como por ejemplo el lenguaje académico, el económico empresarial o el de anuncios publicitarios, los estudios sobre textos turísticos en alemán son escasos. Según Flinz (2011: 156): "Touristische Textsorten sind im Allgemeinen in der Linguistik viel weniger als andere erforscht: selten in kontrastiver Hinsicht und kaum unter dem Aspekt einer kontrastiven Textsortentypologie» (traducción de M. Labarta: Dentro del ámbito de la Lingüística se ha investigado, en general, mucho menos el tipo de texto turístico que el de otros tipos de texto: son escasos los estudios desde el punto de vista contrastivo y raros los realizados desde la perspectiva de una tipología textual contrastiva). Los primeros estudios lingüísticos sobre textos turísticos en alemán datan de los años 70 (Fischer, 1976 y Putschögl-Wild, 1978) y tienen como áreas de interés la formación de palabras, la sintaxis, la estructura de los folletos y la semántica. Posteriormente surge un mayor interés por los textos turísticos y en los últimos años ya encontramos trabajos tanto monolingües de alemán, como contrastivos entre el alemán y otras lenguas, que persiguen diferentes objetivos de análisis lingüísticos. Entre los monolingües podemos destacar los trabajos de Kulzer (2007) y Baldauf-Quilliatre (2010). El primero analiza los aspectos pragmáticos y la forma lingüística de los folletos, así como la 
relación existente entre el texto e las imágenes; el segundo tiene un enfoque de tipología textual y considera al folleto de viaje un tipo de texto publicitario. En cuanto a estudios contrastivos, aunque apenas existen trabajos que se concentren en las dos lenguas, el alemán y el español, sí los hay del alemán y otras lenguas, como el finlandés, el polaco o el italiano, entre otras. Del alemán-finlandés destacamos aquí las investigaciones de Natunen (2001) y de Heimo (2007), que analizan los problemas de la traducción de los prospectos o folletos turísticos. Smykala (2011) por su parte realiza un estudio contrastivo detallado entre folletos turísticos austriacos y polacos. Flinz (2011) analiza de forma contrastiva los textos turísticos de catálogos de viaje en alemán y en italiano. La mayoría de estos estudios contrastivos tiene como objetivo central de investigación la aplicación a la traducción.

El discurso del turismo forma parte de los llamados lenguajes de especialidad, caracterizados por ser el producto de la actividad comunicativa de una comunidad discursiva concreta, en este caso la del turismo. Igual que estableció Pickett (1989) al definir el discurso empresarial, podríamos decir que el lenguaje del turismo es un ergolecto o lenguaje de trabajo, y se caracteriza por tener unas funciones comunicativas y unos conceptos propios, todo lo cual le confiere una identidad estructural y léxico-semántica que lo diferencia de otros lenguajes de especialidad, como puedan ser el económico, el jurídico, el político, etc. Estas funciones comunicativas y conceptos se expresan a través de estructuras verbales recurrentes y a través de un léxico o vocabulario específico. Sin embargo, esto sería una descripción general de lo que constituye el discurso del turismo y haría falta profundizar en cuanto a su especificidad para conseguir una descripción más adecuada de cada género, dentro del abanico de posibilidades textuales que conforman el discurso del turismo. Como apunta Swales (1990), cada comunidad discursiva establece unos géneros escritos $u$ orales a través de los cuales sus miembros se comunican, siendo imprescindible que, además, los identifiquen y reconozcan, con objeto de poder reproducirlos y, añadimos aquí, que el propósito comunicativo se pueda cumplir. Así, la comunidad discursiva del turismo tiene unos géneros propios, cada uno con un objetivo comunicativo y unas funciones concretas que pueden presentar cierta variación según los distintos patrones culturales a los que respondan. Esta variable de las funciones comunicativas es importante a la hora de redactar y de traducir cada uno de estos géneros. Sin embargo, también es importante, para conseguir una buena traducción, considerar con precisión los rasgos discursivos interpersonales de cada lengua origen, con la finalidad de intentar reproducirlos y recrear textos que sean fieles al patrón cultural y lingüístico original. Solo así serán reconocibles como tales y podrán cumplir su función comunicativa. Si no, lo que se consigue es un texto correctamente traducido en cuanto a su gramática y sus funciones comunicativas básicas, pero deficiente en la expresión de aspectos lingüísticos más refinados, como son los rasgos discursivos interpersonales. No tener en cuenta estos rasgos puede dar como resultado que el texto traducido esté reproduciendo el discurso del texto y de la lengua origen, que muy probablemente será diferente al de la lengua meta. El resultado final será el de una traducción que no estará reproduciendo ciertos rasgos de uso y que los lectores reconozcan como propios dentro de un género determinado, y, por tanto, el objetivo comunicativo, la persuasión en el género que 
nos ocupa, puede quedar mermada o deteriorada (Suau Jiménez, 2015). Esto es así, debido a que el lector no identificará ese texto con el modelo genérico y las estrategias discursivas que tiene memorizadas en su lengua. Como consecuencia, la respuesta del lector-comprador potencial de productos o servicios turísticos- puede no ser la deseada debido a estos "errores" de traducción, que se pueden ver como un reflejo del servicio o producto que se les está intentando vender, es decir, con escasa calidad. Por tanto, las diferencias de patrón cultural que aparecen en el discurso interpersonal de las distintas lenguas son un elemento a tener en cuenta en las traducciones especializadas. En estas traducciones de los géneros más comunes del turismo -páginas web hoteleras, páginas web de restaurantes, páginas de promoción de lugares-, entre los que se cuenta la Guía Turística, el objetivo comunicativo es la persuasión, para cuya consecución, la relación entre texto y lector debería reproducir las características discursivas interpersonales a las que los lectores están habituados y reconocen como genéricas.

\section{EL DISCURSO INTERPERSONAL Y SU IMPORTANCIA EN GÉNEROS DE PROMOCIÓN}

¿Qué es el discurso interpersonal o interpersonalidad? Podría definirse como el conjunto de elementos (marcadores y estrategias) que un autor utiliza para expresar autoridad y credibilidad, y para involucrar al lector a través del texto, y así alcanzar el objetivo comunicativo del género. Algunas aproximaciones recientes al análisis del discurso han revisado el concepto de género y han introducido el de constructo social (Fairclough, 1992, 1995), sugiriendo que los textos son parte de los acontecimientos sociales o la forma lingüística en la cual las personas actúan e interactúan socialmente. Tanto un autor como su audiencia son vistos como agentes sociales cuyas acciones no son libres, ya que están socialmente delimitadas o constreñidas. El discurso interpersonal tiene su origen en el concepto de metadiscurso interpersonal (Vande Kopple, 1985; Crismore et al., 2003; Hyland \& Tse, 2004; Hyland, 2005, 2008, por citar a algunos autores), el cual se ha aplicado principalmente a los géneros académicos en lengua inglesa. Hyland \& Tse (2004) y Hyland (2005) sugieren que el (meta)discurso interpersonal es una categoría funcional dentro del discurso, que no se puede dejar de tener en cuenta. A través de él, un autor puede transformar un texto difícil o duro en prosa amable y de lectura más placentera, a la vez que establece un vínculo personal con el lector. Se realiza lingüísticamente a través de verbos en condicional, verbos modales, adverbios, verbos en imperativo, pronombres personales, adjetivos calificativos, etc. según la convención de los distintos géneros, los requisitos de cada disciplina y el trasfondo cultural de cada lengua, todo lo cual impone unas restricciones específicas, dando lugar al patrón metadiscursivo, o conjunto de estrategias y marcadores concretos para cada texto analizado.

A partir de estos estudios, el grupo de investigación Interlae de la universidad de Zaragoza propuso el término interpersonalidad (Lorés-Sanz, Mur-Dueñas \& Lafuente, 2010). Este trabajo y este nuevo concepto incluye el estudio, no solo de géneros académicos, sino también de otros géneros como las reseñas de libros, las popularizaciones digitales, los blogs, los ensayos de alumnos y los informes de evaluaciones, en disciplinas que van desde la economía y la empresa hasta el derecho, pasando por la literatura, la lingüística, la medicina o la

Normas (ISSN: 2174-7245) | 
psicología. Breeze, Gotti y Sancho (2014) también abogan por la interpersonalidad como un concepto paraguas, parcialmente sinónimo de metadiscurso interpersonal, ya que recoge una variedad de géneros y disciplinas que van más allá del ámbito académico. Finalmente, Suau Jiménez (2016), retomando el testigo del concepto interpersonalidad, propone el término discurso interpersonal como concepto que no solo incluye marcadores metadiscursivos, sino también proposicionales o de contenido. Esta propuesta se sustenta principalmente en diferentes estudios realizados con corpus de géneros del turismo, en los cuales son esenciales los marcadores que potencian valores turísticos, representados por realzadores, en forma de adjetivos calificativos de tono positivo, a la vez que marcadores de actitud, que pueden también materializarse a través de adjetivos y/o colocaciones nominales (Mapelli, 2008; Pierini, 2009; Edo-Marzá, 2012, Suau-Jiménez, 2012a, 2012b).

El concepto de discurso interpersonal también puede contemplarse desde el punto de vista del registro (Halliday, 1985), concretamente en la función interpersonal -tenor-, que atiende al modo en que el emisor organiza el propio texto y la forma a través de la cual se relaciona con el receptor. Así pues, el discurso interpersonal hace que el autor de un texto muestre cómo están relacionadas las diferentes partes de un texto y cómo han de ser interpretadas por el lector. Es especialmente importante en géneros profesionales y sociales con un claro propósito de promoción, como ocurre con la Guía Turística, pretendiendo así que el lector no solo descodifique un mensaje, sino también que, como resultado, actúe y se convierta en consumidor de productos o servicios turísticos.

\subsection{Marcadores discursivos interpersonales en alemán y español}

El discurso interpersonal como marco metodológico de análisis lingüístico tiene marcadores muy bien definidos y útiles y otros más discutibles. Han sido estudiados principalmente en los géneros académicos y científicos en inglés (Hyland \& Tse, 2004; Hyland, 2005), y también en alemán. Concretamente, nos basamos en el estudio contrastivo alemán-inglés de Clemen (1998) sobre textos especializados en economía, y en el estudio contrastivo alemán-inglésespañol de Nord (2002) sobre textos publicitarios.

En su análisis de los atenuadores o hedges (Hecken) alemanes del discurso especializado en economía, Clemen (1998) llega a la conclusión de que existen dos tipos de atenuadores discursivos en alemán: atenuadores epistémicos, epistemische Hecken, y atenuadores de motivación interpersonal, interpersonal motivierte Hecken.

La categoría principal de los atenuadores epistémicos, se refiere por una parte a verbos relacionados con el "saber", "creer" y "conocer", etc. Por otra, a otras expresiones de posibilidad, probabilidad y del grado de certeza que tiene el autor sobre lo que está escribiendo, así como sus reflexiones sobre ello. Aquí hace una subdivisión de diferentes subcategorías, como "Verbos epistémicos" (denken, glauben, verstehen, vermuten, annehmen); "Grado de conocimiénto del autor" (angeblich, fraglich vielleicht, offensichtlich); "Hipótesis a través de subjuntivo, Konjunktiv II (wäre günstig, hätte den Vortei); 
"Condicionalidad" (wenn ... wäre, würde ...); "Adversativas y conjunciones restrictivas" (aber, obgleich, obwohl, trotz, ungeachtet), etc.

Los atenuadores de motivación interpersonal no reflejan el conocimiento, sino la actitud o posicionamiento del autor con respecto al texto. Algunas de los tipos que menciona Clemen son "Comentarios del autor de tipo evaluativo" (überraschenderweise, glücklicherweise, wichtig, erfreulicherweise, bedeutsam, bedauerlicherweise, entscheidend, meiner Meinung/Ansicht nach); "Intensificadores" (bestimmt, durchaus, fraglos, freilich, gewiß, klar, natürlich, offensichtlich (wie jedersehen kann), selbstverständlich, sicher, tatsächlich, wirklich, zugegebenermaßen, zweifellos) y "Partículas modales", tan importantes y prácticamente exclusivas en alemán, ya no suelen tener expresiones léxicas paralelas ni en inglés ni en español. Ejemplos de ello serían: nur, ja, denn, doch, eigentlich.

El análisis de Clemen utiliza también el modelo de Hyland \& Tse (2004), que amplía con las correspondientes formas alemanas. Para los realzadores ingleses in fact, e it is clear that propone una larga serie de atenuadores, entre ellos selbsverständlich o zweifellos, etc. Son formas de reafirmar la certeza de una enunciación a través de adverbios o expresiones congeladas. Sin embargo, creemos que los adjetivos calificativos de tono positivo y, a veces, hasta superlativo están utilizados por el autor para dar un matiz de realce, énfasis o modulación subjetiva al contenido proposicional, y por tanto servirían para establecer el vínculo interpersonal con el lector. Aunque esto es discutible y necesitaría más teorización, así los hemos considerado en nuestro análisis. Los marcadores de actitud, tal como los definen ellos, tendrían para nosotros aspectos parecidos a los realzadores de Hyland \& Tse (2004), o sea, serían adverbios y expresiones congeladas tipo sicher, zweifellos, wirklich en alemán (en español, seguro, sin duda alguna, realmente; en inglés sure, really without doubt) Los marcadores de compromiso no admiten dudas, ya que se ejemplifican a través de referencias directas al lector por medio de verbos en imperativo (gehen Sie!, lassen Sie sich verwöhnen!) y referencias apelativas al lector a través del pronombre Sie, muy frecuentes en el discurso alemán. Finalmente, la automención también tiene la función de crear una relación entre autor y lector, y se suele expresar a través del pronombre wir, primera persona del plural en alemán.

Por su parte Nord (2002) analiza los indicadores de la función fática, fundamentales para la persuasión, en un corpus de textos publicitarios en inglés, español y alemán. La función fática es esencial para el discurso interpersonal, ya que, según Jakobson (1960) está orientada a construir el canal de comunicación entre el emisor y el receptor. Su propósito es iniciar, prolongar, interrumpir o finalizar una conversación o bien comprobar si existe algún tipo de contacto. Además de esto, para Nord (2002) la definición y configuración (Ausgestaltung) de la relación social entre emisor y receptor entra también dentro del ámbito de la función fática. Se refiere con ello a la forma de dirigirse a alguien, es decir qué pronombre, título o registro es el más adecuado. Todos estos aspectos ayudan a materializar lingüísticamente la persuasión.

Los marcadores de la función fática son para Nord, por una parte la forma de tratamiento (die Anrede), y por otra, el registro utilizado (Chrystal \& Davy 1969). Respecto al tratamiento, Nord 
llega a la conclusión de que los textos publicitarios en español indican una relación mucho más íntima o de más confianza con el público al que van dirigidos que los textos alemanes, en los cuales se utilizan los pronombres Sie y Ihnen, como formas de tratamiento de "usted". Los textos alemanes marcan claramente una mayor distancia social. Mientras que en los textos españoles se trata al receptor de igual a igual, en los textos alemanes se prefiere una relación más formal. Según Nord (2002:160) esto refleja el uso de las formas de tratamiento de ambas culturas en la vida real. Estamos de acuerdo con Nord en que hay cuestiones que son "kulturspezifisch", es decir, que no dependen de la lengua en sí (como sistema lingüístico) sino de la cultura a la que pertenece.

La principal diferencia respecto al registro informal está en que el alemán usa más partículas modales y marcadores del discurso que el español y que el inglés. Los resultados del análisis muestran que, en el corpus español, el registro informal está indicado por las siguientes marcas (Nord, 2002:160): las elipsis sintácticas, p. ej "La prensa está de acuerdo: un turismo que parece un deportivo. O al revés"; los paréntesis, p.ej.: "La única cosa que no cuestionamos (al menos de momento); las oraciones cortadas; estructuras focalizadas (Fokusstrukturen), p. ej.: "El camino para realizar tus sueños sólo lo marcas tú" y en el léxico, palabras del vocabulario y expresiones que según Diccionario del Español Actual (1999) tienen una connotación coloquial o popular, p. ej.: "Baje de las nubes y toque de pies en Ofiprix". Lo menos frecuente son los marcadores del discurso. En el caso de los textos alemanes, también se encuentran elipsis, p. ej.: "Lieber trocken trinken als trocken feiern", oraciones cortadas y en el léxico, palabras que según el Diccionario Duden-Wörterbuch der deutschen Sprache (1993) son coloquiales (umgangsprachlich), p. ej. Job, loslegen, Geld machen y mal. Además de éstas marcas, aparecen algunas formas coloquiales de contracción: etwa hat's y geht's, La principal diferencia está en el uso de las partículas modales y marcadores del discurso, como por ejemplo ja, doch, einfach, na. En resumen, teniendo en cuenta las formas de tratamiento y el registro utilizados, los textos de promoción españoles analizados combinan las formas de tratamiento de tú con marcas de registro informal para crear una relación de confianza con el público al que va dirigida la promoción. Mientras que en los textos en alemán, el tratamiento es de "usted" y son las partículas modales las que juegan un papel esencial para la función fática y la persuasión.

Finalmente, la categoría de marcadores de compromiso, correspondería en el análisis de textos publicitarios o de promoción de Nord a los Adressierungsmarker y Kontaktindikatoren, es decir, marcadores o indicadores de contacto. Los resultados de su análisis muestran que el tipo de ejemplo que predomina en este caso son las referencias directas al lector por medio de verbos en imperativo (2002:156) (entdecken Sie!, Profitieren Sie von....), que cumplen claramente una función apelativa, al igual que en el tipo de texto turístico, objetivo de nuestro análisis.

En cuanto a los marcadores de la función interpersonal del español, los estudios iniciales también se aplicaron a géneros académicos (Ferrari y Gallardo, 2003; Beke, 2005; MurDueñas, 2007; Vázquez y Giner, 2009, por citar solo a algunos autores). El énfasis de estos 
estudios se pone en los atenuadores o matizadores discursivos, quizá por ser estos muy representativos en los artículos de investigación y géneros afines, pero también en marcadores de actitud y realzadores. Todos estos marcadores, sin embargo, pertenecen a la voz del autor (White, 2003; Hyland, 2008), predominante en todos los géneros académicos, por ser el autor en quien recae el peso de la convicción o persuasión propia del artículo de investigación, basadas en la autoridad que ha de desprender este género. La aproximación a géneros noacadémicos desde el discurso interpersonal o desde paradigmas cercanos se centra en estudios de Suau Jiménez (2006, 2012a, 2012b), Pierini (2009) o Mapelli (2008), todos ellos aplicados al ámbito del turismo y a los géneros de promoción como páginas web institucionales o guías turísticas. Estas autoras hacen constar la importancia de ciertos marcadores, como los realzadores, que se revelan como fundamentales en la transmisión de valores turísticos predeterminados -lo único, lo espectacular, lo moderno, lo confortable, lo grandioso- , y, a la vez, prototípicos de estos géneros. Su realización lingüística se basa en adjetivos calificativos de tono positivo, a veces superlativo, así como en sintagmas nominales, principalmente ("las monumentales carrozas", "las extraordinarias vistas", "el tradicional confort de las habitaciones", "el incomparable paisaje"). También se dan los atenuadores/matizadores y los marcadores de actitud, todos ellos también como parte de la voz del autor "podemos elegir una habitación u otra", "se puede optar por comida casera o internacional"/ "claramente, es la mejor visita", "es especialmente importante reservar con antelación"), pero en menor medida que los realzadores.

Es de destacar la escasa ocurrencia de marcadores asociados a la voz del lector, tales como los marcadores de compromiso o del lector, con o sin pronombres -tú o usted- ("no puedes perderte el festival"), o los directivos en forma de imperativos ("vaya a la taquilla si no quiere perder tiempo"), que, sin embargo, sí son importantes en inglés (Suau Jiménez, 2012b), al igual que ocurre en alemán. Esto es debido posiblemente al sustrato cultural del discurso interpersonal, que se alinea con los usos de cortesía propios de cada lengua, y que en español no contempla una implicación excesiva del lector en el mensaje, al contrario de lo que ocurre en inglés o en alemán. Resumiendo, se observan importantes diferencias entre los marcadores en alemán y en español en los mismos géneros del turismo y en ambas lenguas originales.

\section{OBJETIVO E HIPÓTESIS}

El objetivo de nuestro estudio es el de analizar los marcadores que constituyen el discurso interpersonal en alemán y español como lenguas originales, y compararlos con los del alemán como lengua traducida en el género Guía Turística, para observar si en esta última variedad se han considerado y traducido adecuadamente los rasgos interpersonales que prescribe este género y esta lengua. Aventuramos que, posiblemente, no se habrán tenido en cuenta, tal como se ha observado en anteriores trabajos (Suau Jiménez, 2015), con lo cual la consecución de la función persuasiva, propia de la Guía Turística, puede haber sido dañada. Las implicaciones para la traducción son, por lo tanto, muy importantes. 


\section{METODOLOGÍA}

La metodología que hemos seguido en este estudio parte del modelo metadiscursivo interpersonal de Hyland \& Tse (2004) y Hyland (2005), adaptado a los géneros del turismo según el concepto de interpersonalidad antes mencionado (Suau Jiménez, 2012b, 2016). Asimismo, nos hemos basado en el trabajo contrastivo de Clemen (1998) para el análisis concreto del discurso interpersonal en alemán. El corpus piloto del que da cuenta esta investigación se ha extraído de folletos turísticos impresos sobre la ciudad de Valencia, recogidos de la Oficina de Turismo del Ayuntamiento de Valencia (Touristlnfo) y de la Ciudad de las Artes y las Ciencias (CAC), en español como lengua original y en alemán como lengua traducida, además de folletos turísticos impresos de la ciudades de Berlín y de Colonia para el alemán como lengua original. Contiene los tres bloques mencionados: textos en alemán como lengua original, textos en español como lengua original y textos en alemán como lengua traducida, con un total de alrededor de 24.000 palabras.

\section{RESULTADOS Y ANÁLISIS}

Estos son los resultados del análisis de los tres bloques del corpus antes mencionado: alemán como lengua original, español como lengua original y alemán como lengua traducida del español.

\subsection{Discurso en textos turísticos en alemán como lengua original}

\section{Porcentajes}

\begin{tabular}{|l|l|l|l|l|l|}
\hline $\begin{array}{l}\text { Total } \\
\text { palabras }\end{array}$ & Atenuadores & Realzadores & $\begin{array}{l}\text { Marcadores de } \\
\text { actitud }\end{array}$ & $\begin{array}{l}\text { Marcadores de } \\
\text { compromiso }\end{array}$ & Automención \\
\hline 5.995 & $0,66 \%$ & $7,8 \%$ & $1,40 \%$ & $3,6 \%$ & $0,40 \%$ \\
\hline
\end{tabular}

Ejemplos

\begin{tabular}{l}
\hline Atenuadores \\
\hline "Auch den grandiosen Blick über den großen Hafen und den Jadebusen sollten Sie nicht \\
verpassen". \\
"Hier können Sie die Tiere in freier Wildbahn beobachten. Abfahrtsort ist Dangast". \\
"An Bord gibt es ausreichende Verpflegung, und in den Fischgründen der Nordsee erwartet Sie \\
hoffentlich ein guter Fang". \\
"Deutschland kann Ihnen jeden Wunsch erfüllen". \\
\begin{tabular}{|l|}
\hline Realzadores \\
\hline "Wir freuen uns, Sie in einer der lebendigsten Großstäte Europas willkommen zu heißen".
\end{tabular}
\end{tabular}


"Stadt und Region gewährleisten heute eine optimale Errreichbarkeit zu Land, zu Wasser und zu Luft."

"Als Messe und Kongresszentrum steht Köln ganz oben".

"Werden Sie Augenzeuge einer der extremsten Welten, deren Geburtsstunde am Ende der letzten Eiszeit lag: Das Wattenmeer!"

"Auch den grandiosen Blick über den großen Hafen und den Jadebusen sollten Sie nicht verpassen. "Der Kölner Dom ist eine Kathedrale der Superlative".

"Nicht weniger beindruckend ist seine Grundfläche: Die Gesamfläche des Doms beträgt 145 Meter, das Querhaus ist 86 Meter breit".

Marcadores de actitud

"Nicht von ungefähr wird Deutschland oft als das Land der Dichter und Denker bezeichnet".

"Nicht zuletzt deshalb, steht Köln für Lebensfreude pur".

"Der Fläche nach belegt die Domstadt nach Berlin und Hamburg sogar den dritten Platz".

"Mit über 1000 Zügen täglich ist der Kölner Hauptbahnhof sogar der verkehrsreichste

Eisenbahnknotenpunkt Deutschlands“.

Marcadores de compromiso/Pronombres del lector

"Wir freuen uns, Sie in einer der lebendigsten Großstäte Europas willkommen zu heißen".

"Werden Sie Augenzeuge einer der extremsten Welten, deren Geburtsstunde am Ende der letzten Eiszeit lag: Das Wattenmeer!“

"Gehen Sie im Wattenmeerhaus auf eine einzigartige Entdeckungsreise und lassen Sie sich in unserer $2500 \mathrm{~m}^{2}$ großen Ausstellung verzaubern".

\begin{tabular}{|l|}
\hline Automención \\
\hline "Wir freuen uns, Sie in einer der lebendigsten Großstäte Europas willkommen zu heißen". \\
"Mit weiteren Fragen, wenden Sie sich einfach an uns". \\
"Wir wünschen Ihnen einen angenhemen Aufenthalt in Köln". \\
\hline
\end{tabular}

\subsection{Discurso interpersonal de los textos en español como lengua original}

\section{Porcentajes}

\begin{tabular}{|l|l|l|l|l|l|}
\hline Total palabras & Atenuadores & Realzadores & $\begin{array}{l}\text { Marcadores } \\
\text { de actitud }\end{array}$ & $\begin{array}{l}\text { Marcadores } \\
\text { de } \\
\text { compromiso }\end{array}$ & Automención \\
\hline 10.865 & $0,42 \%$ & $2,82 \%$ & $0,06 \%$ & $0,04 \%$ & $0,04 \%$ \\
\hline
\end{tabular}

Ejemplos

Atenuadores

(22) "Valencia puede ser una ciudad de tópicos".

(23) "Son claves para tratar de explicar lo que no se puede explicar con palabras".

(24) "Aconsejamos al amigo lector que seleccione si opta por visitar todo un barrio, o..."

(25) "Todo esto se puede complementar con la visita a la Ciudad de las Ciencias". 
El discurso interpersonal en la guía turística... | Francisca Suau y María Labarta

\begin{tabular}{|l|}
\hline Realzadores \\
\hline (26) "Valencia ha experimentado una significativa transformación en los últimos años". \\
(27) "El nuevo Palacio de Congresos...representativa de la arquitectura más vanguardista...". \\
(28) "Llegar por una emocionante ruta al Santuario de la Balma en Zorita.... \\
(29) "Conocer la serena belleza de los parques naturales del Prat de Cabanes..". \\
\hline
\end{tabular}

\begin{tabular}{|l|}
\hline Marcadores de actitud \\
\hline (30) "Se caracteriza por un clima suave, típicamente mediterráneo". \\
(31) “... claves para tratar de explicar lo que, en realidad, no se puede contar con palabras". \\
(32) “...están transformando definitivamente el perfil urbano".
\end{tabular}

\begin{tabular}{|l|}
\hline Marcadores de compromiso \\
\hline (33) “... no deje de tener en cuenta la Lonja de la Seda”. \\
(34) "... al amigo lector que seleccione..."
\end{tabular}

\section{Automención}

(35) "Aconsejamos al amigo lector que seleccione..."

(36) "Vamos a sugerirles unos cuantos de los museos y monumentos..."

\subsection{Discurso interpersonal de los textos en alemán como lengua traducida del español}

Porcentajes

\begin{tabular}{|l|l|l|l|l|l|}
\hline Total palabras & Matizadores & Realzadores & $\begin{array}{l}\text { Marcadores } \\
\text { de actitud }\end{array}$ & $\begin{array}{l}\text { Marcadores } \\
\text { de } \\
\text { compromiso }\end{array}$ & Automención \\
\hline 6.009 & $0,6 \%$ & $4,2 \%$ & $0,12 \%$ & $1,18 \%$ & $0,06 \%$ \\
\hline
\end{tabular}

\section{Ejemplos}

\begin{tabular}{|l|}
\hline Atenuadores \\
\hline (37) "Was kann man in Castellon unternehmen?" \\
(38) “...und selbst im Winter warm, und selbst im Winter kann man sonnenbaden." \\
\hline
\end{tabular}

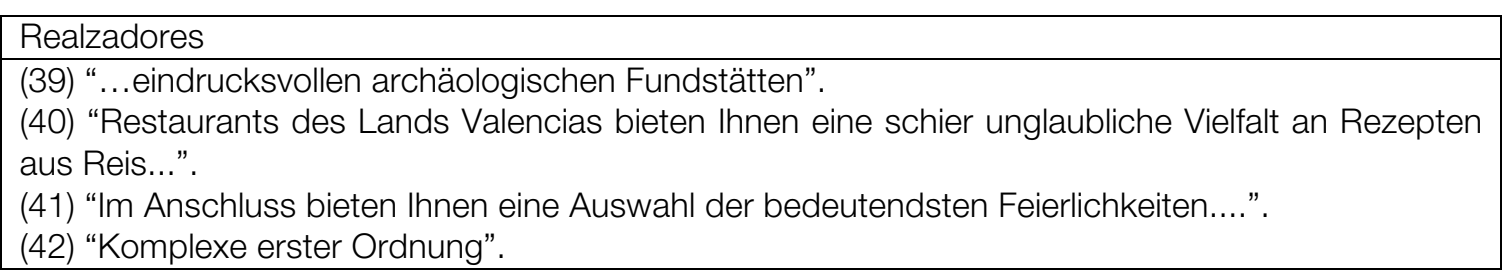

Marcadores de actitud
(43) "Zur Wahl stehen Mittelmeerkreuzfahrten oder sogar die Möglichkeit, Boote mit oder ohne
Bootsführer anzumieten".


Marcadores de compromiso/Marcadores del lector

(44) Restaurants des Lands Valencias bieten Ihnen eine schier unglaubliche Vielfalt an Rezepten aus Reis..."

(45) “...fast ganzjährig für Sie geöffnet”.

\begin{tabular}{|l|}
\hline Automención \\
\hline (46) "Im Anschluss bieten wir Ihnen eine Auswahl der bedeutendsten Feierlichkeiten...." \\
\hline
\end{tabular}

Si comparamos los textos escritos en la lengua original podemos observar que mientras el uso de atenuadores es solo algo más elevado en alemán que en español, sí existe una gran diferencia en cuanto al uso de los realzadores. Éstos son más del doble en los textos alemanes que en los españoles.

El uso de marcadores de compromiso, de cierta importancia en alemán (3,6 \%), es escaso, sin embargo, en español (0,04\%). También la automención es más utilizada en los textos escritos en alemán (proporcionalmente 10 veces más), ya que presenta un 0,04\% en español y un $0,40 \%$ en alemán. $Y$, por último, los marcadores de actitud, también presentan un porcentaje doble en alemán (0’12\%) respecto al español (0’06\%).

Todas las categorías analizadas tienen, con diferencia, mayor representación en los textos alemanes como lengua original. Nos queda por ver ahora a cuál de estos patrones se asemeja más el texto traducido español-alemán.

Si comparamos los textos traducidos al alemán con los de alemán como lengua original observamos grandes diferencias en casi todas las categorías. Tan solo las cifras respecto a los atenuadores son similares, siendo esto la excepción. El uso de marcadores de actitud es mucho menor en los textos traducidos $(0,12 \%)$; el número de realzadores también se reduce a casi la mitad $(4,2 \%)$ frente a $7,8 \%$ en alemán original; los marcadores son apenas un tercio $(1,18 \%)$ de los que se usan en alemán lengua origen $(3,6 \%)$ y finalmente, la automención es seis veces menor en las traducciones $(0,06 \%)$.

Sin embargo, sí encontramos mayor similitud entre los textos traducidos y los textos en español lengua original. Con una ligera diferencia en los valores correspondientes a automención, los marcadores de actitud coinciden. Los marcadores de compromiso, apenas inexistentes en español $(0,04 \%)$, tienen cierta presencia en los textos traducidos al alemán $(1,16 \%)$, pero menos de un tercio del tanto por ciento que aparece en los textos alemanes originales (3,6\%). En cuanto a los realzadores, el valor de 4,2\% se acerca más al del español original $(2,82 \%)$ que al alemán original, casi el doble (7,8\%). En general, podemos concluir diciendo que los textos alemanes traducidos tienen más en común con los textos originales españoles que con los de la lengua origen alemán en lo que a metadiscurso interpersonal se refiere. 


\section{DISCUSIÓN Y CONCLUSIONES}

A la vista de los resultados del análisis, podríamos decir que existen diferencias considerables entre el discurso alemán y el español, ambos como lengua original, en la Guía Turística. El discurso alemán tiene muy en cuenta, por una parte, los marcadores de compromiso o marcadores del lector, especialmente en cuanto a la pronominalización a través del Sie, un claro elemento que realiza la función interpersonal de Halliday y que establece un vínculo directo con el lector y lo involucra en el discurso. Es decir, se trata de un discurso rico y potente en cuanto a su función interpersonal. Por el contrario, el discurso español es muy diferente, con un bajo número de elementos discursivos interpersonales, comparado con el patrón original propuesto en el modelo de Hyland \& Tse (2004) y Hyland (2005), a excepción de los realzadores, un marcador algo confuso, como ya indicamos anteriormente, ya que, aunque lo utiliza el autor para expresar al lector unas cualidades connotativas no directamente medibles ni objetivables, pero importantes para el objetivo comunicativo de este género, no crea una relación clara con el tenor ya que no se refiere directamente a él/ella, como sí ocurre con el Sie alemán.

El discurso interpersonal en español es más escaso si lo comparamos con el alemán; es dependiente del contenido proposicional, parte esencial del significado, ya que sus elementos más característicos son los realzadores, materializados a través de adjetivos calificativos en su mayoría, que transmiten valores como lo tradicional, lo inolvidable, lo histórico, etc. Pero incluso esta categoría tiene mayor y más diversa representación en los textos alemanes. Por otra parte, los realzadores que aparecen en los textos alemanes son de diferentes tipos (sustantivos, adjetivos, frases completas) como muestran los siguientes ejemplos:

"in der Schönheit ihrer Schlösser und in der Vielseitigkeit ihrer kulturellen Angebote"

"Bei besonderen Aktionen während der Ferienzeit stehen Spaß, Ausdauer und Geschicklichkeit auf dem Programm."

"Keine andere deutsche Stadt hat - umgerechnet auf die Zahl ihrer Einwohner - so viele Kneipen, aber auch so viele erstklassige Restaurants."

Mientras que en los textos españoles en su mayoría se trata de adjetivos calificativos positivos:

1. "Las fiestas de la Comunidad Valenciana son coloristas, abiertas, hechas para celebrarse en la calle".

2. "El visitante puede desde conocer la emblemática Ciudad de las Artes y las Ciencias de Valencia, recrearse con la programación de Castellón cultural, vivir experiencias inolvidables...."

3. "Los restaurantes de la Comunidad Valenciana ofrecen una increíble variedad de recetas elaboradas con arroz". 
Por otra parte, en el alemán como lengua traducida, hemos observado que, aunque es menor el uso de los realzadores, en su mayoría se trata de adjetivos, como vemos en los siguientes ejemplos. Por tanto, echamos de menos en los textos traducidos la diversidad de tipos de marcadores de esta categoría que nos ofrecen los textos en alemán como lengua original.

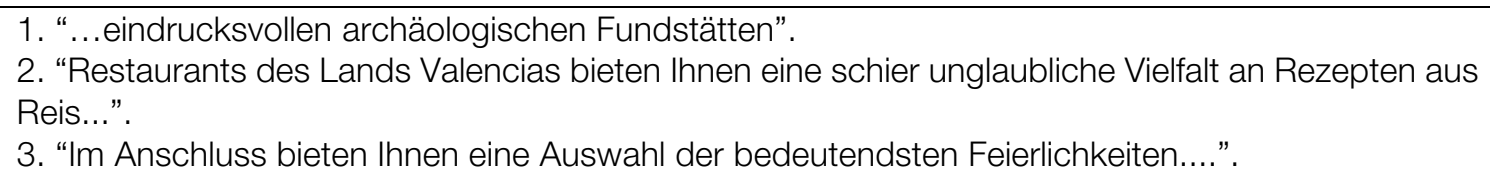

El discurso interpersonal en alemán como lengua traducida es bastante más reducido en cuanto a la cantidad de marcadores, si lo comparamos con el alemán como lengua original, ya que, a excepción de los matizadores o atenuadores, no utiliza suficientemente los elementos característicos de la lengua original, tales como los marcadores de actitud, de compromiso y de automención.

En conclusión, diríamos pues, que, en efecto, parece que no se ha tenido en cuenta el discurso interpersonal del alemán como lengua meta al traducir este corpus del género Guía Turística, como así lo indican las tablas en las que hemos desglosado el patrón interpersonal resultante, según las categorías propuestas. Al contrario, se observa una reproducción del discurso interpersonal español en la traducción, ya que solo hay una categoría en la que éstos coinciden: los marcadores de compromiso o marcadores del lector. En el resto de los marcadores hallados en la traducción, con respecto a la lengua de la que se traduce (el español), los valores son mucho más similares a los del español que a los del alemán como lengua original. En cuanto a la automención, otra categoría importante en el discurso turístico alemán, tampoco se ha tenido muy en cuenta y su representación es escasa. Es decir, la traducción reproduce las categorías o marcadores más importantes del discurso turístico en español pero no las de la lengua meta, es decir, la de los lectores a quienes va dirigida la Guía Turística, el alemán.

Podemos plantearnos la pregunta de hasta qué punto se pueden considerar estas deficiencias un error en la traducción. Es difícil de contestar, si bien lingüísticamente hablando y para ser coherentes con toda la base teórica de nuestro estudio, en el cual hemos tratado de exponer la importancia del discurso interpersonal como elemento necesario para que los géneros llamados de promoción cumplan su propósito persuasivo, se podría decir que sí es en cierta medida un error de traducción. Podemos argumentar, según los resultados de este estudio, que no se ha tenido en cuenta el sustrato cultural y los usos de cortesía de la lengua meta, el alemán, que prescribe un discurso en el cual son muy importantes ciertos elementos que establecen la relación interpersonal con el lector.

Como consecuencia de esta carencia discursiva y según el concepto de texto como constructo social (Fairclough, 1992), que es parte de un acontecimiento social (el turismo), los 
textos turísticos traducidos al alemán que hemos analizado podrían no influir de forma adecuada y completa en las acciones de los actores sociales, aquí turistas potenciales.

\section{BIBLIOGRAFÍA}

BECK, Klaus (2008): "Neue Medien - alte Probleme? Blogs aus medien- und kommunikationsethischer Sicht" en ZERFASS, Ansgar et al. (ed.): Kommunikation, Partizipation und Wirkungen im Social Web. Grundlagen und Methoden: Von der Gesellschaft zum Individuum. Köln: von Halem, 62-77.

BEKE, Rebeca (2005): "El metadiscurso interpersonal en artículos de investigación", Signos, 38(57), 7-18

BREEZE, Ruth, GOTTI, Maurizio y Carmen SANCHO (2014): Interpersonality in Legal Genres, Bern, Peter Lang.

CALVI, Maria Vittoria (2006): Lengua y comunicación en el español del turismo, Madrid, Arco Libros

CALVI, Maria Vittoria (2010): Los géneros discursivos en la lengua del turismo: una propuesta de clasificación, Ibérica, 19, 9-32.

CALVI, Maria Vittoria (2016): Guía de viaje y turismo 2.0: los borrosos confines de un género, Ibérica, 31,15-38.

CRISMORE, Avon, MARKANEN, Raija y Margaret STEFFENSEN (1993): "Metadiscourse in persuasive writing", Written Communication, 10 (1), 39-71.

CLEMEN, Gudrun (1998): Hecken in deutschen und englischen Texten der Wirtschaftskommunikation: eine kontrastive Analyse, Universität Siegen, Dissertation.

Diccionario del Español Actual (1999): SECO, Manuel, ANDRÉS, Olimpia y RAMOS, Gabino (eds.): Diccionario del Español Actual. 2 Vol. Madrid, Aguilar.

Duden-Das große Wörterbuch der deutschen Sprache (1993): DROSDOWSKI, Günther et al. (eds). Duden - Das große Wörterbuch der deutschen Sprache. 7 Vol. Mannheim, Dudenverlag.

DURÁN MUÑOZ, Isabel (2011): El trabajo ontoterminográfico aplicado a la traducción de textos del turismo de aventura (español, inglés, alemán): fases de preparación, elaboración y edición, Málaga, Servicio de Publicaciones de la Universidad de Málaga.

EDO MARZÁ, Nuria (2012): "Páginas web privadas e institucionales: el uso de la adjetivación en un corpus inglés-español de promoción de destinos turísticos”, en Sanmartín Sáez, Julia (ed.), Discurso Turístico e Internet, Madrid, Iberoamericana/Vervuert, 51-80.

FISCHER, Ludwig (1976): "Dienstbarkeit und Subversion. Skizze zu einer Erörterung über die Funktion von Kunstmitteln in Reise-katalogen" en Fischer, Ludwig, Hickethier, Knut y Ritha, Karl (eds.), Gebrauchsliteratur. Methodische Uberlegungen und Beispiel-analysen, Stuttgart, 203-244.

FAIRCLOUGH, Norman (1992): Discourse and Social Change, Cambridge, Polity Press.

FAIRCLOUGH, Norman (1995): Critical Discourse Analysis, Harlow, Longman.

FLINZ, Carolina (2011): "Mikrostrukturelle Eigenschaften touristischer Fachtexte: ein Vergleich deutscher und italienischer Reisekataloge für die DaF- Didaktik", Studi Linguistici e Filologici Online, 9.2, 155-180

GALLARDO, Susana (2009): "La estructura ilocutiva y la distinción entre géneros discursivos" en Ciapuscio, Guiomar (ed.), De la palabra al texto, Estudios lingüísticos del español, Buenos Aires, Eudeba, 129-162.

GAST, Volker (2008): "Modal Particles and Context Updating - the Functions of German ja, doch, wohl und etwa" en Heinz Vater, Ole Letnes (eds), Modalverben und Grammatikalisierung . Trier: Wissenschaftlicher Verlag, 153-177

HALLIDAY, Michael A.K. (1978): Language as Social Semiotic, London, Arnold.

HALLIDAY, Michael A.K. (1994): An Introduction to Functional Grammar, 2nd edition. London: Edward Arnold.

HEIMO, Taina (2007): "Vergleich von deutsch- und finnischsprachigen Flyern im Bereich-Tourismus Probleme in der übersetzung", Beiträge zur Fremdsprachenvermittlung, 46, 113-126

HYLAND, Kenneth (1994): "Hedging in Academic Writing and EAP Textbooks", English for Specific Purposes, 13/3, 239-256.

HYLAND, Kenneth y Polly TSE (2004): "Metadiscourse in Academic Writing: A Reappraisal", Applied Linguistics, 25/2, 156-177. 
HYLAND, Kenneth (2005): "Stance and engagement: a model of interaction in academic discourse", Discourse Studies, 7 (2), 173-192.

HYLAND, Kenneth (2008). "Disciplinary voices. Interactions in research writing", English Text 1, 1, 5-22.

JAKOBSON, Roman (1960): "Linguistics and Poetics" en Thomas Sebeok (ed), Style in Language. Cambridge, MA: M.I.T. Press, 350377.

JANICH, Nina (2005): Werbesprache. Ein Arbeitsbuch, Tübingen, Narr Verlag.

KRANICH, Svenja (2009): "Epistemic modality in English popular scientific articles and their German translations. trans-kom", Zeitschrift für Translationswissenschaft

and Fachkommunikation 2 [1]: 26-41.

KRANICH, Svenja (2011): "To hedge or not to hedge. The use of epistemic modal expressions in popular science in English texts, English-German translations and German original texts", Text \& Talk 31 [1]: 77-99.

KRANICH, Svenja (2016): Contrastive Pragmatics and Translation. Evaluation, Epistemic Modality and Communicative Style in English and German. Amsterdam, John Benjamins.

LABARTA POSTIGO, Maria (1996): Linguistische Eigenschaften und Verständlichkeit von Software-Dokumentationen, Eine anwendungsorientierte Untersuchung, Frankfurt a. M., Peter Lang.

LABARTA POSTIGO, Maria / ORTEGA OLIVARES, Jenaro (2001): "La reiteración" en: Graciela Vázquez. (ed.). Guía Didáctica del Discurso Académico Oral. 1ed. Madrid, Edinumen, 1, 5784.

LABARTA POSTIGO, Maria (ed.) (2005): Approaches to Critical Discourse Analysis, Valencia, Servei de Publicacions de la Universitat de Valencia.

LABARTA POSTIGO, Maria (2007): "Los matizadores lingüísticos en alemán: Análisis de textos especializados en Turismo", Transformaciones Culturales: Debates de la Teoría, la Crítica y la Lingüística, 1, 1-15, Buenos Aires, Universidad de Buenos Aires.

LORÉS-SANZ, Rosa, MUR-DUEÑAS, Pilar y Enrique LAFUENTE (2010): Constructing Interpersonality: Multiple Perspectives on Written Academic Genres, Newcastle, Cambridge Scholar Publishings.

MAPELLI, Giovanna (2008): "Las marcas de metadiscurso interpersonal de la sección 'turismo' de los sitios web de los ayuntamientos", en Calvi, Maria Vittoria, Mapelli, Giovanna y Javier Santos (eds), Lingue, culture, economía: comunicazione e pratiche discorsive, Milano, Franco Angeli, 173-190.

MUR-DUEÑAS, Pilar (2010): "Attitude markers in business management research articles: a crosscultural corpus-driven approach", International Journal of Applied Linguistics, 20, 1, 50-72.

NATUNEN, Martina (2001): Probleme beim Ubbersetzen von Fremdenverkehrsprospekten (Finnisch-Deutsch) en Liefländer-Koistinen, Luise (ed.), Aus eigener Werkstatt - eine Auslese aus Unterricht und For-schung der deutschen Abteilung, Universität Joensuu, Veröffentlichungen des Instituts für interkulturelle Kommunikation, 89-114.

NORD, Christiane (1997): Translating as a Purposeful Activity: Functionalist Approaches Explained, Manchester: St Jerome.

NORD, Christiane (2002): "Über-Reden durch AnReden Die phatische Funktion als Mittel zum Appell in englischen, spanischen und deutschen Werbetexten" en Linguistics and Translation Studies - Translation Studies and Linguistics, 145-167

NORD, Christiane (2003): Kommunikativ handeln auf Spanisch und Deutsch. Einübersetzungsorientierter, korpusgestützter Sprach- und Stilvergleich, Wilhelmsfeld, Gottfried Egert Verlag.

PICKET, David (1989): "The sleeping giant: investigations in business English”. Language International, 1, 1, 5-11.

PIERINI, Patricia (2007): "Quality in Web Translation: An Investigation into UK and Italian Tourism Web Sites", The Journal of Specialized Translation, Issue 8

[http://www.jostrans.org/issue08/art_pierini.php, fecha de consulta: 15 mayo 2014].

PIERINI, Patricia (2009): "Adjectives in Tourism English on the Web. A corpus-based study". CÍRCULO de Lingüística Aplicada a la Comunicación, 40/2009, 93-116.

PUTSCHOGL-WILD, Anna Monika (1978): Untersuchungen zur Sprache im Fremdenverkehr, Frankfurt a. M., Peter Lang.

SANTULLI, Francesca (2007): "II discorso procedurale come tratto distintivo della guida turistica" en Garzone, Giuliana y Rita Salvi (eds.), Linguistica. Linguaggi specialistici. Didattica delle lingue, Roma, CISU, 227-240. 
SMYKALA, Marta (2011): "Stilistische Merkmale der Textsorte Tourismusprospekt aus kontrastiver Sicht (Deutsch-Polnisch). Oder: Wie lobt man das eigene Land"en Zofia Bilut- Homplewicz, Angnieszka Mac, Marta Smykala, Iwona Szwed (eds.), Text und Stil, Frankfurt a. M., 221-232.

SUAU JIMÉNEZ, Francisca (2012a): "Páginas web institucionales de promoción turística: el uso metadiscursivo interpersonal en inglés y español”, en Sanmartín Sáez, Julia (ed.), Discurso Turístico e Internet, Madrid: Iberoamericana/Vervuert, 125-154.

SUAU JIMÉNEZ, Francisca (2012b): "El turista 2.0 como receptor de la promoción turística: estrategias lingüísticas e importancia de su estudio", PASOS. Revista de Turismo y Patrimonio Cultural, 6, 4, Número Especial, 144152.

SUAU JIMÉNEZ, Francisca (2016): "What can the construction of stance and engagement voices in traveller forums and tourism promotional websites bring into a cultural, cross-generic and disciplinary view of interpersonality?", Ibérica, 31, 199-220.

VANDE KOPPLE, William J. (1985): "Some Explanatory Discourse on Metadiscourse", College Composition and Communication, 36, 82-93.

VÁZQUEZ, Ignacio y Diana GINER (2009): "Writing with conviction: The use of boosters in modelling persuasión in academic discourses". Revista Alicantina de Estudios Ingleses 22, 219-23.

VESALAINEN, Marjo (2001): Prospektwerbung. Vergleichende rhetorische und sprachwissenschaftliche Untersuchungen an deutschen und finnischen Werbematerialien, Frankfurt a.M., Peter Lang.

WHITE Peter (2003): "Beyond modality and hedging: a dialogic view of the language of intersubjective stance", Text 23(2), 2594-2598. 